\title{
eJRIEPS
}

Ejournal de la recherche sur l'intervention en éducation physique et sport

$17 \mid 2009$

Varia

\section{Style d'un passeur de haut niveau en volley-ball}

\section{Serge Eloi}

\section{OpenEdition}

\section{Journals}

Édition électronique

URL : http://journals.openedition.org/ejrieps/5606

DOI : 10.4000/ejrieps.5606

ISSN : 2105-0821

Éditeur

ELLIADD

\section{Référence électronique}

Serge Eloi, « Style d'un passeur de haut niveau en volley-ball », eJRIEPS [En ligne], 17 | 2009, mis en ligne le 01 avril 2009, consulté le 18 mars 2021. URL : http://journals.openedition.org/ejrieps/5606 ; DOl : https://doi.org/10.4000/ejrieps.5606

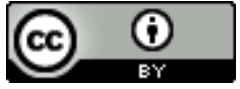

La revue eJRIEPS est mise à disposition selon les termes de la Creative Commons Attribution 4.0 International License. 
Style d'un passeur de haut niveau en volley-ball

\section{Serge Eloi}

Université Paris 12 - Val de Marne

GÉDIAPS (Groupe d'Études «Didactique et Intervention dans le Domaine des Activités Physiques et Sportives).

\section{Résumé}

Si tous les spécialistes (entraîneurs, joueurs, dirigeants) s'accordent à reconnaître le rôle prépondérant du passeur dans le niveau de performance d'une équipe de volley-ball, il n'existe paradoxalement, que très peu de travaux ou littérature disponibles sur ce thème. Notre objectif a consisté à identifier les principaux éléments pris en compte par les passeurs de haut niveau dans la gestion des situations de haute complexité inhérentes à leur fonction. Pour recueillir les verbalisations de notre passeur, nous avons utilisé la méthode de l'entretien d'explicitation (Vermersch, 1994 ; Vermersch \& Maurel, 1997). II est discuté de la pertinence du choix de ce type d'entretien pour caractériser le style du passeur (Clot \& Faïta, 2000). À la suite d'une analyse technologique du rôle de distributeur, il apparaît que 3 axes permettent de rendre compte de l'activité du passeur de volley-ball. Les axes spatio-temporel, stratégico-tactique et technico-technique s'avèrent du même coup renseigner la triade de l'activité (Clot, 1999) qui fait référence à l'objet de la tâche, au rapport aux autres ainsi qu'au rapport au sujet lui-même. L'exploitation des verbatim permet de définir à l'intérieur du genre spécifique de passeur, le style particulier d'un passeur. Cet article présente une étude de cas. L'outil construit offre finalement la perspective de son utilisation systématique pour permettre la comparaison avec d'autres passeurs.

Si tous les spécialistes (entraîneurs, joueurs, dirigeants) s'accordent à reconnaître le rôle prépondérant du passeur dans le niveau de performance d'une équipe de volley-ball, il n'existe paradoxalement, que très peu de travaux ou littératures disponibles sur ce thème (Coulibaly, 1984). Par ailleurs, l'usage montre que la plupart du temps, lors de la formation, c'est la relation passeur - attaquants qui est principalement travaillée (Simonet, Hippolyte, \& Petit, 1990). L'entraînement est alors essentiellement orienté vers les techniques spécifiques (Schmitt, 1996). Cependant, l'analyse du système de contraintes dans lequel le passeur est imbriqué (Éloi, 2001) montre que cette relation (passeur- 
attaquants) n'est pas la seule à pouvoir déterminer le niveau de performance d'une équipe sur le plan offensif. II semble notamment que le passeur, plongé dans des situations cycliques d'opposition, soit amené à planifier ses interventions. Notre objectif a consisté à identifier les principaux éléments pris en compte par le passeur de haut niveau dans la gestion des situations de haute complexité inhérentes à sa fonction. Cet article rend compte d'une étude de cas : le cas de Jules.

\section{Le rôle de passeur au volley-ball}

1. 1. Analyse de l'activité volley-ball

Le volley-ball est un sport collectif qui oppose 2 équipes disposées de part et d'autre d'un filet. Une équipe est composée de 6 joueurs. Chaque équipe dispose de 3 touches de balle pour tenter de faire tomber le ballon dans le camp adverse. Les 6 joueurs sont répartis en deux lignes. Une ligne de 3 avants qui ont le droit d'attaquer (smasher) près du filet. Une ligne de trois arrières qui doivent prendre leur appel derrière la ligne des $3 \mathrm{~m}$ pour pouvoir attaquer. La ligne de contre est constituée des 3 avants.

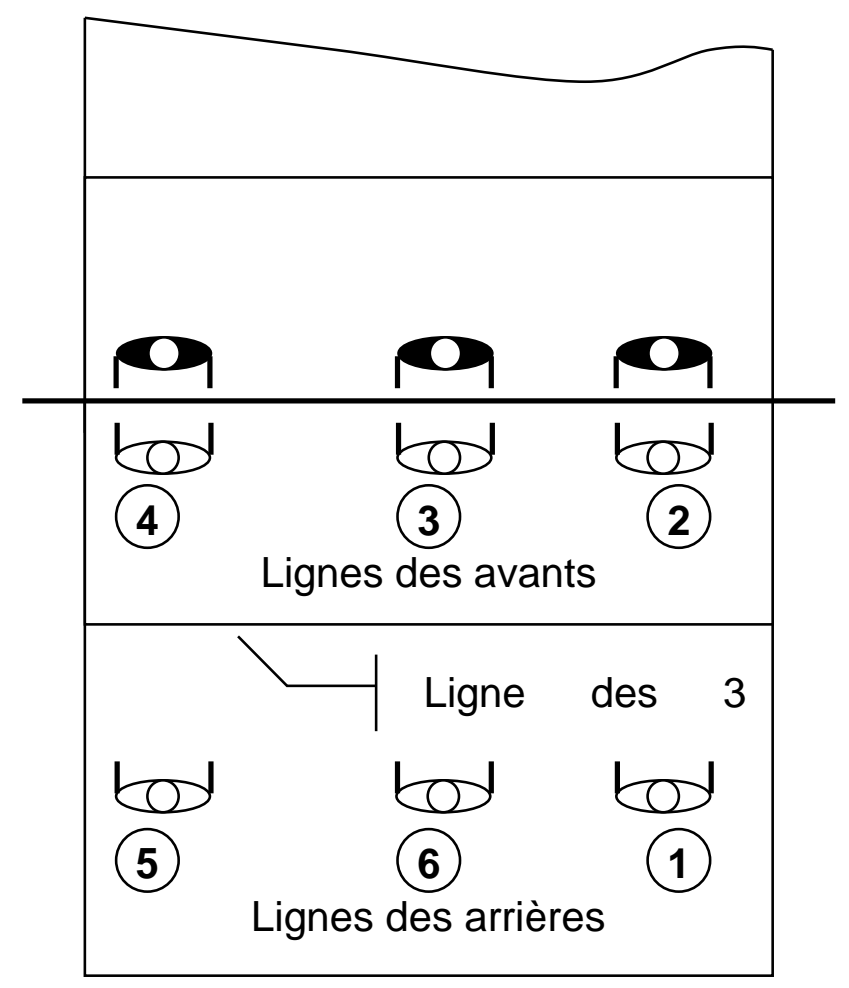

Figure 1. Position des joueurs sur le terrain 
Classiquement, un échange débute par un service c'est-à-dire une mise en jeu qui parvient de l'autre côté du filet, dans le camp adverse. Les adversaires effectuent une réception de service (1ère touche de balle) qui est destinée à un joueur particulier dont le rôle est de distribuer le jeu. On le nomme le passeur.

Ce passeur fait une passe au joueur de son choix (2e touche de balle). Le joueur destinataire de la passe tente de marquer le point en smashant la balle dans le camp adverse (3e touche de balle). II nous faut maintenant approfondir cette description sommaire pour comprendre les enjeux liés à ce rôle de distributeur.

1. 1. 1. Essence des sports collectifs, essence du volley-ball

Pour définir l'essence du volley-ball, c'est-à-dire le rapport fondamental qui fonde cette activité (Éloi \& Uhlrich, 2001), nous faisons le constat que, comme pour d'autres sports collectifs, ce jeu requiert deux cibles et un seul projectile (Deleplace, 1983). Le ressort du jeu est donc fondé sur le fait qu'il faut être en possession du ballon pour pouvoir tenter d'atteindre la cible adverse. Ainsi, nous pouvons constater que ce dispositif engendre une véritable lutte entre protagonistes adverses pour entrer en possession du ballon. II s'agit de «gérer simultanément ou bien la conservation de la balle pour son équipe et la conquête de la cible opposée, ou bien la conquête de la balle et la défense de sa propre cible » (Bonnefoy, Lahuppe, \& Né, 1997, p. 16). C'est donc cette lutte qui constitue pour nous, l'essence même des sports collectifs. De par son caractère collectif, ce combat met en scène opposition-coopération, continuité-rupture, mais ces particularités ne sont que la conséquence du mécanisme initié préalablement par la forme contradictoire des enjeux respectifs.

\section{1. 2. Caractéristiques spécifiques du volley-ball}

Dans les règles officielles de 2005, on peut lire que « le but du jeu est d'envoyer le ballon par-dessus le filet afin qu'il retombe dans le terrain opposé et d'empêcher cette même action de la part des adversaires »(FIVB, 2005). Ainsi, la cible est l'aire délimitée de l'autre côté du filet, aire dans laquelle évoluent les adversaires. II n'y a donc pas d'interpénétration des espaces de jeu. II découle d'une analyse faite par ailleurs (Éloi \& Uhlrich, 2001) que la spécificité du volley-ball est principalement contenue dans le fait que la lutte pour entrer en possession du ballon est différée (si on la compare à d'autres activités comme le rugby où le combat est immédiat). De cette latence dans le combat (nécessité d'attendre que la balle revienne) découle le principe suivant: de la qualité de mon renvoi dépend la qualité du renvoi de mon adversaire. Cette réflexion nous permet de définir la logique contradictoire du volley-ball. Elle peut être conçue comme un mécanisme particulier, une dialectique spécifique, un enjeu décisif. Plus précisément, nous la 
décrirons comme une loi de fonctionnement et de développement inhérente à cette activité sportive. Cette loi peut s'énoncer ainsi : au volley-ball, il s'agit de générer de la continuité dans son propre camp pour mieux créer la rupture dans le camp adverse. C'est sur la base de cette relation dialectique que va se structurer la relation spécifique des deux équipes qui s'affrontent dans le cadre du volley-ball. Ce détour théorique nous permet de montrer que le rôle du passeur va se situer à l'intersection de ces deux notions contradictoires que sont continuité et rupture.

1. 2. Le passeur au cœur d'un système de contraintes Ainsi donc, le passeur se trouve à la jonction de deux phases de jeu distinctes :

- La première qui a pour objectif de contrôler les tentatives offensives de l'adversaire et donc de générer de la continuité dans son propre camp.

- La seconde qui a pour objectif d'organiser les alternatives d'attaques de ses partenaires et donc de provoquer la rupture dans le camp adverse.

Cette position particulièrement centrale du passeur dans l'organisation du jeu au sein de l'équipe lui confère une grande responsabilité liée à son rôle d'« interface ». Dans ses mains se joue, comme par délégation, le schème du duel des deux équipes (Récopé, 1996, 2002).

\section{3. Différentes sources de complexité}

La mise en difficulté que le passeur est amené à subir de ses réceptionneurs d'une part (via la qualité du service de l'adversaire) et sa volonté de positionner au mieux ses attaquants d'autre part (en fonction des combinaisons d'attaques annoncées), révèlent la complexité du dispositif dans lequel il se trouve imbriqué.

\section{3. 1. Le passeur comme interface d'un milieu changeant}

Cette complexité est renforcée par le caractère cyclique des situations d'opposition. En effet, si l'équipe qui est en réception remporte l'échange, elle procède à une rotation dans le sens des aiguilles d'une montre. Le joueur qui était avant droit se retrouve en position d'arrière droit et va donc servir. Ces rotations ont comme conséquence un changement du rapport d'opposition au rythme des échanges remportés ou perdus. Pour le passeur, sa position sur le terrain au moment du service n'est pas la même. Mais plus encore, sa ligne d'attaque et la ligne de contre adverse changent également. II faut alors gérer lors d'un même set, douze rapports d'opposition différents (chaque équipe tourne à tour de rôle), successifs (ils se succèdent dans un ordre déterminé) et répétitifs (un set correspond à environ à 2,5 rotations complètes de l'équipe). 
1. 3. 2. Le passeur face à une ligne de contre

Si comme nous l'avons décrit avant, l'un des facteurs de complexité réside dans la variation des situations d'opposition, il est patent qu'un autre aspect concerne la gestion de chacune de ces positions. II s'agit de déterminer le schéma offensif propre à mettre en difficulté le contre adverse. II faut donc prendre en compte les particularités de cette ligne de contreurs, mais également leur éventuelle capacité à s'adapter aux choix d'attaques effectués précédemment. C'est donc le jeu du chat et de la souris qui prévaut ici. Les contreurs adverses tentent de réduire l'incertitude liée au type d'attaque que le passeur adverse va choisir. Le passeur tente de masquer le plus longtemps le choix qu'il fera en fin de compte. Il y a donc un véritable intérêt à déterminer les informations perçues, les connaissances mobilisées où celles qui sont construites par le passeur pour prendre en compte l'ensemble de ces contraintes (Macquet, 2001).

L'analyse du jeu dévoilée plus avant permet de comprendre les responsabilités multiples endossées par le passeur. Elle nous amène aussi à mieux appréhender le rôle central et prépondérant que ce joueur peut jouer au sein de son équipe.

\section{Méthodologie de la recherche}

À la lumière de ce qui précède, il paraît légitime de se poser la question de savoir comment le passeur de haut niveau organise l'ensemble des informations qu'il peut recueillir avant et pendant le jeu pour aboutir à un choix de passe.

\section{1. Question de recherche}

Nous ferons l'hypothèse que cette décision, bien que prise en milieu complexe, est reliée à un cadre d'analyse structuré. Notre objectif consiste donc à décrire ce cadre et à identifier les stratégies déployées de façon spécifique par le passeur.

\section{2. Cadre théorique}

Notre cadre d'analyse se positionne à l'intersection des trois domaines théoriques suivants: la psychologie du travail sous l'angle de la clinique de l'activité, la psycho phénoménologie et la technologie des Activités Physiques Sportives et Artistiques (APSA). Cette imbrication des champs conceptuels pose la question du choix de la méthode.

2. 2. 1. Problématique du choix de la méthode

Comment envisager de renseigner des aspects aussi intimes que ceux qui concernent la prise de décision dans l'action sans se référer directement aux protagonistes de ces choix ? Si la technique de l'entretien semble s'imposer, il reste à déterminer le type d'entretien le plus approprié pour être en mesure de répondre à la question posée. La référence à la clinique de l'activité (Clot, 1999) et à la psycho-phénoménologie 
(Vermersch, 1999 ; Vermersch, 2002a) nous engage à une discussion à propos du choix de la méthode. Quel est, de l'entretien d'explicitation ou des autoconfrontations (autoconfrontation simple, autoconfrontation croisée, instruction au sosie), celui qui est le plus apte à renseigner l'activité décisionnelle ? Le débat concernant la singularité de l'action (Barbier, 2000) prend ici tout son sens. Comme le souligne Clot, « le réel de l'expérience ne se touche pas facilement du doigt ». II s'agit donc de "réunir certaines conditions pour se frayer un accès à la singularité de l'action» (Clot, 2000, p. 54). II propose de faire du concept d'activité dirigée l'unité élémentaire d'analyse en psychologie du travail. Nous adhérons à l'idée que l'activité est triplement dirigée : par la conduite du sujet, au travers de l'objet de la tâche et vers l'activité des autres (Clot, 1999). Cette triade (sujet, tâche, autrui) qui constitue l'unité élémentaire d'analyse de l'activité est le lieu d'un conflit permanent qui oppose ces trois points de vue et contribue à mettre en scène les contradictions desquelles une voie de développement fini par se dégager. Loin d'être une entité figée, cette triade est pour reprendre la formule de Clot, « une cellule vivante ». Elle est le cœur d'un dispositif de sélection, d'orientation mais aussi de conflits et donc d'inhibition des activités. Agir, c'est donc autant s'orienter que réfuter des voies possibles. II faut donc assimiler que l'analyse de l'action consiste à comprendre comment les autres voies possibles sont écartées au profit de celle qui est empruntée. Pour Clot, « l'objet de la connaissance est moins l'activité que le développement de l'activité et ses empêchements » (Clot, 2004, p. 31). Du coup, l'objectif poursuivi par la clinique de l'activité s'écarte sensiblement de nos préoccupations. Alors même que le cadre théorique adhère précisément aux questions épistémologiques qui concernent la prise de décision, nous prenons la mesure de la différence d'objectif relatif à notre propre projet scientifique. Pour la clinique de l'activité, c'est l'activité et son développement qui sont au centre du débat. Notre propos est différent. Nous voulons identifier ce qui confère de l'efficacité à l'activité décisionnelle. Si la clinique de l'activité se propose « d'étudier les organisateurs et l'organisation de la transformation de l'activité »(Clot, 2004, p. 31), nous cherchons ici à faire le recueil des modes de prises de décisions qui conduisent à la réussite ou à l'échec de l'action. Nous ne nous plaçons pas dans le cadre d'une démarche longitudinale qui prend en compte l'évolution d'un collectif de travail sur une longue période. Nous voulons faire le bilan des stratégies de prises de décisions déployées aujourd'hui par les passeurs du plus haut niveau. De ce fait, les méthodes d'autoconfrontation habituellement utilisées pour analyser le développement de l'activité ne paraissent pas se justifier dans le cadre de notre recherche. II nous semble en effet préférable d'aborder le problème par le versant des opérations plutôt que par celui de l'activité. Nous faisons référence ici à la théorie de 
l'action développée par Leontiev qui détermine trois niveaux d'analyse. Celui de l'activité qui est orientée par les motifs d'agir, celui de l'action qui est orientée par un but et celui des opérations qui sont orientées par les conditions pratiques de réalisation des actions (Léontiev, 1972). Pour lui, il est acquis que trois types d'opérations organisent l'action : les opérations d'exécution, les opérations de contrôle et les opérations d'orientation. Si les opérations d'exécution et de contrôle sont visibles et donc évaluables, les opérations d'orientation de l'action sont inaccessibles à l'observation. Or, pour Leontiev, la réussite de l'action est dépendante de la qualité des opérations d'orientation. II apparaît alors que renseigner les opérations d'orientation devient nécessaire.

\section{2. 2. L'entretien d'explicitation}

La discussion nous engage à opter pour l'entretien d'explicitation. Non pas que ce choix dénie la pertinence du cadre théorique d'analyse de l'activité. Bien au contraire, nous le verrons plus loin, nous pourrons renseigner les catégories de sujet, tâche et autrui qui forme la triade élémentaire de l'activité dirigée. Toutefois, nous prenons le parti d'aborder l'activité par son élément le plus fin: les opérations d'orientation. Et pour atteindre cet objectif, il semble bien que la méthode de l'entretien d'explicitation soit particulièrement adéquate (Vermersch, 2002b). Cette technique d'entretien permet en effet au sujet de « re-contacter » le moment d'action vécu. L'entretien d'explicitation est tout entier tourné vers la verbalisation d'une action effectivement accomplie, effectivement vécue lors d'une occurrence singulière par la personne qui en parle (Vermersch et Maurel, 1997). À partir de la description du vécu du sujet exprimé sur la base de «comment il a fait », notre objectif est d'accéder au couple « objectivation de l'action / subjectivité du sujet » pour mettre en relation la logique (non observable) du sujet et la logique (observable) du jeu. Pour cela, l'interviewer utilise des techniques proches de la programmation neurolinguistique (PNL). L'objectif est de mettre le sujet en état d'évocation en utilisant son canal sensoriel privilégié (visuel, auditif ou kinesthésique). II est donc nécessaire de déterminer au début de l'entretien de quel sens il s'agit afin d'utiliser lors de l'entretien, un vocabulaire en adéquation avec ce canal sensoriel spécifique. II devient ensuite possible d'obtenir des verbalisations proches des sensations éprouvées lors de l'action. C'est ce que l'on appelle l'état d'évocation (Vermersch, 2002a). Dans la lignée des travaux d'autres auteurs (Gouju, 2005 ; Mouchet, 2005), nous avons donc utilisé l'entretien d'explicitation pour dévoiler au travers de ce que Vermersch nomme «la pensée privée » (Vermersch, 2002a), les stratégies de prises de décisions du passeur de volley-ball. Se pose alors la question de l'exploitation des données recueillies. 


\section{Analyse des données}

Si comme nous l'avons vu précédemment, la question de la nature du corpus n'offre pas d'alternative, se pose subséquemment le problème de la manière dont ce recueil peut être exploité. Comment passer des données en première personne (ce que nous livre le sujet) à des données organisées sur la base d'une analyse aussi rigoureuse que possible ? Doiton pour traiter les verbatim utiliser la méthode des fenêtres attentionnelles (Gouju, 2002) ? Doit-on tenter de reconstruire le scénario du jeu (Mouchet, 2003) ? Les données verbales recueillies à l'aide d'une méthode d'entretien bien identifiée ne peuvent-elles être traitées que d'une seule et même façon ? La spécificité de l'activité étudiée ne nous engage-t-elle pas à définir un cadre d'analyse adapté à ce contexte précis ?

II nous est apparu que les données à traiter sont indépendantes de la méthode avec laquelle elles sont recueillies. Que par ailleurs, il est nécessaire de retravailler les catégories d'analyse du discours en fonction du contexte de l'action. Enfin, qu'il est incontournable de procéder à de nombreux allers-retours entre les verbatim et les catégories de données pour aboutir à un cadre d'analyse cohérent permettant de structurer le discours issu de l'entretien. C'est à cette condition que l'on peut reconstruire l'activité du sujet pour la rendre compréhensible par tous. L'analyse technologique de l'activité a donc été à l'origine du cadre conceptuel de traitement des données. Mais les mises à l'épreuve successives de ce cadre nous ont amenées à procéder à différentes restructurations. II nous a semblé qu'une cohérence optimale a été atteinte lorsque l'analyse technologique du rôle de passeur et l'analyse de l'activité du sujet (au sens de la clinique de l'activité) pouvaient fusionner de façon homogène. Cette cohérence a pris sens lorsqu'il est devenu possible à l'aide des trois axes issus de l'analyse technologique des actions du passeur de renseigner en même temps la triade de l'activité.

\section{1. Détermination des catégories d'analyse}

Comme nous l'avons développé plus haut, la mise en difficulté que le passeur est amené à subir des réceptionneurs d'une part, et de sa volonté de positionner au mieux ses attaquants d'autre part, témoigne de la complexité du dispositif dans laquelle il se trouve imbriqué. Le concept de catégories d'analyse a montré ses limites dans la mesure où il fallait pouvoir utiliser un outil d'évaluation dynamique et réutilisable pour d'autres passeurs. En effet, dans une situation similaire, tel passeur va réagir plutôt de cette façon et un autre différemment. II nous est apparu que l'idée d'utiliser des axes était particulièrement adaptée à la catégorisation de nos distributeurs de jeu. Il s'agissait alors de positionner sur un axe déterminé un curseur exprimant les options retenues par le sujet étudié. Ce dispositif ne contredit en rien les exigences d'exhaustivité et d'exclusivité 
propre à toute grille d'analyse. Ainsi, chaque mécanisme de prise de décision explicité par un passeur doit trouver une place sur chacun des axes (exigence d'exhaustivité). De la même manière, tout mode de distribution ne doit pouvoir être affecté qu'à un seul emplacement sur chaque axe (exigence d'exclusivité). L'analyse des verbatim consiste donc à discuter du positionnement du curseur sur chacun des axes en prenant appui sur les verbalisations du sujet.

3. 2. Analyse technologique et détermination des axes d'évaluation

S'il s'avère illusoire de vouloir simplifier toute complexité, il semble néanmoins possible de la modéliser (Le Moigne, 1999 ; Morin \& Le Moigne, 1999). La détermination des axes évoqués plus avant nous permet de structurer l'espace des possibles. C'est à leur description que nous allons procéder maintenant. II apparaît alors que nous pouvons réorganiser les modes de gestion du passeur selon trois axes :

- Un axe qui va du spatial au temporel en référence à l'organisation des alternatives d'attaques que peut produire le passeur.

- Un axe qui va du stratégique au tactique et qui permet de caractériser les conditions de prise en compte de l'opposition.

- Un axe dédié à la technique qui spécifie le mode de centration du passeur lors de son action de passe.

C'est dans une relation dialectique que le système des axes a été mis à l'épreuve de plusieurs entretiens. Les caractéristiques de chaque nouveau passeur nous ont conduites à affiner la détermination des degrés spécifiques de chaque axe.

\section{2. 1. L'axe spatio-temporel}

Comme l'ont montré Fournier et Petit (1977) au volley-ball les alternatives d'attaques sont organisées par emboîtement de la composante temporelle et de la composante spatiale. En effet, ce sont les différentes combinaisons d'appels de balle qui permettent d'élever au plus au point l'incertitude de la défense adverse (ligne de contre) concernant le lieu et le moment où se produira véritablement l'attaque. On comprendra que pour un passeur de haut niveau, les considérations liées aux temps d'attaques, aux espaces d'attaques et à la combinaison de ces deux dimensions soit une préoccupation fondamentale.

La dimension temporelle

Pour expliquer les enjeux liés à la dimension temporelle, le lecteur doit assimiler qu'il existe trois temps d'attaque au volley-ball.

Le 1er temps implique qu'un contreur qui a la volonté de contrer un attaquant de 1er temps doit sauter en même temps que lui. La balle sort des mains du passeur et est 
attaquée immédiatement (le ballon parcourt moins d'un mètre). On appelle ce type d'attaque une courte.

Le 2e temps d'attaque se situe au moment où l'attaquant de premier temps retombe au sol. Ainsi, la conséquence déterminante de cette synchronisation tient au fait qu'un contreur qui saute sur un 1er temps ne peut pas ressauter sur un 2e temps puisqu'il touche le sol au moment où la balle est frappée par l'attaquant de 2 e temps. Ici se situe le point névralgique de la dimension temporelle. Elle implique que le contreur central doit faire des choix, des options. II ne peut-être sur toutes les attaques.

Le $3 e$ temps d'attaque ne présente que peu d'intérêt stratégique. À haut niveau, il fait état de l'incapacité d'une équipe à s'organiser selon un schéma offensif établi. La balle du passeur est tellement haute qu'elle ne contient plus aucune incertitude pour le contre adverse. Il y a alors présence de deux voire trois contreurs et les chances pour l'attaquant de faire le point sont réduites.

La dimension spatiale

Elle fait référence aux différents lieux d'attaques. Ceux-ci peuvent se trouver plus ou moins éloignés du passeur dans le sens de la largeur. Une autre dimension spatiale fait référence au sens de la profondeur. En effet, le joueur qui attaque peut se trouver dans l'espace avant (joueurs avants pouvant prendre leur appel à l'intérieur de la zone des 3 mètres) ou dans l'espace arrière (joueurs arrières qui doivent prendre leur appel derrière la ligne des 3 mètres). On peut donc dire que les attaques peuvent venir de n'importe quel endroit à l'intérieur d'une zone de 9 mètres de large (espace de passage du ballon au dessus du filet) et 3 mètres de profondeur.

La dimension spatio-temporelle

Pour complexifier la tâche des contreurs, les alternatives d'attaques proposées sous forme de combinaisons coordonnent les dimensions temporelles et spatiales. Ces combinaisons sont l'héritage du développement historique du volley-ball. Celles dont l'efficacité s'est avérée particulièrement redoutable se sont vues donner un nom. Ainsi on peut entendre parler de la croix, la fausse croix, un intervalle, un piston... Si ces combinaisons sont particulièrement efficaces ce n'est pas parce que la composante temporelle surprend l'adversaire. Ce n'est pas non plus parce que la composante spatiale égare les contreurs. Ce qui confère cette efficacité à ces combinaisons, c'est une dimension supplémentaire. C'est leur coordination spatio-temporelle. II se réalise dans ces schémas offensifs une fusion des deux dimensions qui oblige les défenseurs à faire les options parfaites pour espérer les endiguer. 
L'axe prendra donc en compte l'ensemble des alternatives spatio-temporelles d'attaque allant des options purement spatiales aux options purement temporelles en passant par la combinaison des deux dimensions. Pour positionner notre passeur sur cet axe, il faudra donc se référer à ses verbalisations pour déterminer sa tendance la plus marquée.

\section{2. 2. L'axe stratégico-tactique}

La référence à une opposition ou à un combat détermine un but implicite : celui de la victoire sur l'adversaire. Depuis toujours, «l'art de la guerre » décrit différents niveaux dans l'analyse du rapport d'opposition (Coutau-Bégarie, 2003 ; Rosinski, 1950). Nous avons décliné ces niveaux du stratégique au tactique. Cet axe se propose donc de positionner chaque passeur selon qu'il montre une tendance à être plutôt stratège ou plutôt tacticien. Nous avons défini quatre degrés distincts sur l'axe stratégique - tactique.

La dimension stratégique

Nous proposons de définir le niveau stratégique en référence à ce qui est connu à l'avance, à ce qui a fait l'objet d'une préparation d'avant match. La démarche stratégique englobe donc l'ensemble des décisions menées en dehors de l'action sur le terrain. Ces opérations peuvent relever autant de la connaissance de l'adversaire que de l'assimilation de ces connaissances par les futurs protagonistes. En d'autres termes, la stratégie comprend aussi bien la prise d'information préalable à l'action que l'édification des plans qui en découle.

La dimension stratégico-tactique

Entre la fin et le début de chaque set, l'entraîneur et/ou le capitaine peuvent décider des modifications nécessaires à apporter au jeu. Que ce soit sur le plan de l'adaptation des systèmes ou sur celui de la modification du 6 de départ, chaque équipe dispose de quelques minutes pour concevoir son avenir. Dans cette situation, nous avons quitté la sphère purement stratégique. Bien que nous ne soyons pas encore dans le jeu, la prise en compte du dispositif adverse nous rapproche de la problématique tactique.

La dimension tactique

Nous voilà dans le jeu proprement dit. Un échange vient de se finir. Un autre va débuter. La pression temporelle est présente. Le passeur doit prendre en compte la ligne de contre adverse et annoncer les schémas d'attaques à ses partenaires. L'imminence du service confère à cette situation une dimension tactique importante. De la pertinence du schéma offensif choisi dépend souvent l'issue de l'échange.

La dimension tactico-technique

Maintenant, la balle est en jeu. II s'agit de prendre en compte l'adversaire pour tenter d'échapper au système défensif qu'il présente. Pour le passeur, la tâche revêt de multiples 
facettes : analyser la qualité de la réception, percevoir si tous les joueurs participant au schéma offensif sont disponibles, discerner le jeu des contreurs adverses, faire un choix en conséquence et délivrer une passe la plus parfaite techniquement.

Chaque degré de cet axe se rapporte à des moments relativement identifiés du jeu. Les propos du passeur peuvent donc être ainsi référencés à ces moments spécifiques.

3. 2. 3. L'axe technico-technique

Cet axe envisage parmi les typologies possibles une gradation allant d'un rapport au jeu égocentré, c'est à dire centré sur la technique gestuelle utilisée lors de la passe, à un rapport au jeu exocentré, c'est-à-dire centré sur les adversaires. Un intermédiaire à ces deux extrêmes pourrait être représenté par un rapport au jeu centré sur les partenaires. Ainsi, nous avons pu relever, lors d'études préliminaires, que les passeurs pouvaient revêtir des focalisations différentes. C'est ce qui explique que l'axe se déploie d'une référence technique égocentrée (technique «pure») à une référence technique exocentrée (technique «pour »). Les différents échelons de l'axe vont donc exprimer différents degrés de détachement du passeur par rapport à son action.

Technique «pure »

Ici il est fait référence à des verbalisations qui sont centrées sur la réalisation technique de la passe. On trouve dans les propos du passeur toutes les indications qui ont trait à son ressenti lors de ce geste. Les informations sont surtout de l'ordre du kinesthésique : attitude, position du corps, fléchissement des jambes, orientation des épaules, hauteur de ses mains, placement des mains, sensations dans les doigts... Le passeur se focalise sur les conditions qui lui permettent de réaliser sa passe. II fait abstraction du contexte de jeu pour se recentrer sur ses sensations. Sa technique est égocentré.

Un partenaire

Au-delà de sa technique, un passeur peut se focaliser sur l'un de ses partenaires. Bien qu'il puisse avoir jusqu'à quatre attaquants à sa disposition, il arrive qu'il privilégie plus particulièrement une relation avec l'un d'entre eux. Un passeur est assujetti à ce type de focalisation quand il cherche à déterminer les dispositions de son partenaire. S'il se présente dans un bon timing d'attaque. S'il est disponible, si leur connivence peut s'exprimer pleinement.

\section{Les partenaires}

Cette focalisation met en exergue la prise en compte par le passeur de l'ensemble des partenaires. Ce qui fait l'objet d'une attention particulière c'est la synchronisation des différents déplacements des partenaires les uns par rapport aux autres. Le passeur se concentre alors sur leurs positionnements, leurs déplacements. Son point de focalisation 
concerne donc la coordination de l'ensemble des partenaires qui participent au schéma offensif.

Adversaire(s) direct(s)

Cette centration renvoie à la prise en compte de la position du contre adverse. Si la plupart du temps, c'est le contreur central qui représente la menace principale, dans certaines situations, des focalisations peuvent s'effectuer sur des couples de joueurs. C'est la raison pour laquelle dans cette catégorie, nous prenons en compte la possibilité que l'obstacle à franchir s'exprime par des observations relevant de plusieurs contreurs. Le passeur qui est sur ce type de focalisation fait référence à la présence, au retard ou à l'absence de contre. II relève des indices sur la position du ou des contreurs adverses. II exprime les sensations qu'il ressent par rapport à la nature même de l'obstacle qui se dresse de l'autre côté du filet.

Technique «pour»

La prise en compte des adversaires peut se trouver exacerbée dans un jeu de manipulation. Dans ce cas de figure, le passeur relate comment il s'y prend pour leurrer son adversaire. Les descriptions portent ici sur les moyens techniques utilisés pour manipuler l'information. Le passeur utilise alors des techniques particulières de placement des mains, de mouvement des poignets (pompage du ballon) pour orienter ses adversaires dans une voie alors qu'il a choisi d'en emprunter une autre. En fait, il offre à voir ce qu'il va faire pour finalement faire autre chose. La « technique pour » consiste donc pour le passeur à se livrer totalement, à rendre une situation évidente aux yeux de l'adversaire, pour finalement jouer à l'inverse. Lors des entretiens, les passeurs qui font référence à ce type de focalisation décrivent ce que doivent croire leurs adversaires et comment ils manipulent la balle pour arriver à leurs fins.

\section{3. L'entretien}

\section{3. 1. Protocole des entretiens}

L'entretien a été réalisé par une spécialiste de l'entretien d'explicitation qui est aussi formatrice agréée par le GREX 1. Le protocole de début d'entretien a été standardisé. Son originalité tient au fait que nous y avons inclus une clause particulière. En effet, dans un premier temps nous avons demandé au passeur de choisir une action où il a été particulièrement à son avantage. Dans une deuxième partie de l'entretien, nous lui avons demandé de choisir une action où il estimait que son intervention n'avait pas été

\footnotetext{
${ }^{1}$ GREX : Groupe de Recherche sur l'Explicitation.
} 
satisfaisante. L'entretien se déroulait donc en deux périodes. L'une centrée sur une action positive du passeur et l'autre sur une action négative.

\section{3. 2. Apport de la méthode des contrastes}

Cette idée pouvait paraître saugrenue au début de la recherche. Pourquoi prendre en compte une action négative puisque «pour analyser les compétences, il faut analyser l'action efficace » (Pastré, 2002, p. 11). II nous est apparu que nous pouvions aussi renseigner l'activité des passeurs par ce que nous appelons la méthode des contrastes. Au-delà de la distinction classique entre tâche prescrite (ce qui doit être fait), et activité (ce qui se fait) Clot suggère que « ce qui se fait, et que l'on peut considérer comme l'activité réalisée, n'est jamais que l'actualisation d'une des activités réalisables dans la situation où elle voit le jour »(Clot, Faïta, Fernandez, \& Scheller, 2002, p. 18). Pour parvenir à faire ce qu'il va faire, le sujet est l'objet d'un conflit qui l'oppose à ses propres limites, au réel de ce qu'il faut faire, mais aussi aux autres. II y a donc, au-delà de l'activité réalisée, toutes celles qui sont envisagées, rejetées, empêchées ou contrariées. Dans le cadre de notre recherche, la valeur de l'activité qui échoue réside dans le fait qu'elle dévoile l'activité réalisée qu'il n'aurait pas fallu faire, mais en même temps, les activités possibles qui n'ont pas été réalisées. La valeur de la défaillance, c'est qu'elle dévoile des pans de l'activité auxquels on ne peut accéder sous l'angle de l'action réussie. La méthode des contrastes permet donc de comprendre pourquoi ce qui est réussi l'est et en quoi le conflit permanent qui détermine l'activité peut conduire à l'action qui échoue.

3. 4. Le genre et le style du passeur : un volume défini par trois axes Comment définir les particularités d'un passeur ? Comment s'est-il approprié les clés du métier pour exécuter la partition du jeu avec sa sensibilité personnelle ? Comment montrer qu'au-delà d'un rôle similaire d'une équipe à l'autre (celui de passeur), il existe des interprétations singulières ? Pour répondre à cet ensemble de questions, il nous faut revenir sur les concepts de genre et de style. Rappelons tout d'abord que le genre est une façon de réaliser l'activité. Ce concept décrit un répertoire constitué des actes, des conduites, des manières de faire, répertoire qui s'est forgé au cours du développement historique de l'activité et de son milieu d'expression. C'est à travers le genre que les pairs se jugent et s'évaluent. Être passeur, c'est mobiliser un genre particulier au sein de l'ensemble des joueurs de volley-ball. Néanmoins, il est indubitable que des variantes apparaissent d'un passeur à l'autre. Elles naissent de la contrainte de s'adapter à la situation particulière qui se présente ici et maintenant et de la « sensibilité » de chaque sujet. De ce fait, le genre présente toujours une stabilité transitoire. Les retouches et ajustements nécessaires au déroulement de l'activité engagent (obligent) à la création de 
styles individuels. Les mises à l'épreuve de l'activité par la confrontation au réel placent alors le sujet dans une perspective de réinterprétation du genre. « Le style participe au genre auquel il fournit son allure. Les styles sont le retravail des genres en situation, et les genres, du coup, le contraire d'états fixes. Mieux, ils sont toujours inachevés » (Clot \& Faïta, 2000, p. 15). Les retouches permanentes apportées par le sujet concourent donc à faire évoluer le genre. Le genre « passeur de volley-ball » de 2009 est différent du genre «passeur de volley-ball » de 1980. C'est la raison pour laquelle le style peut être défini comme une « métamorphose » du genre.

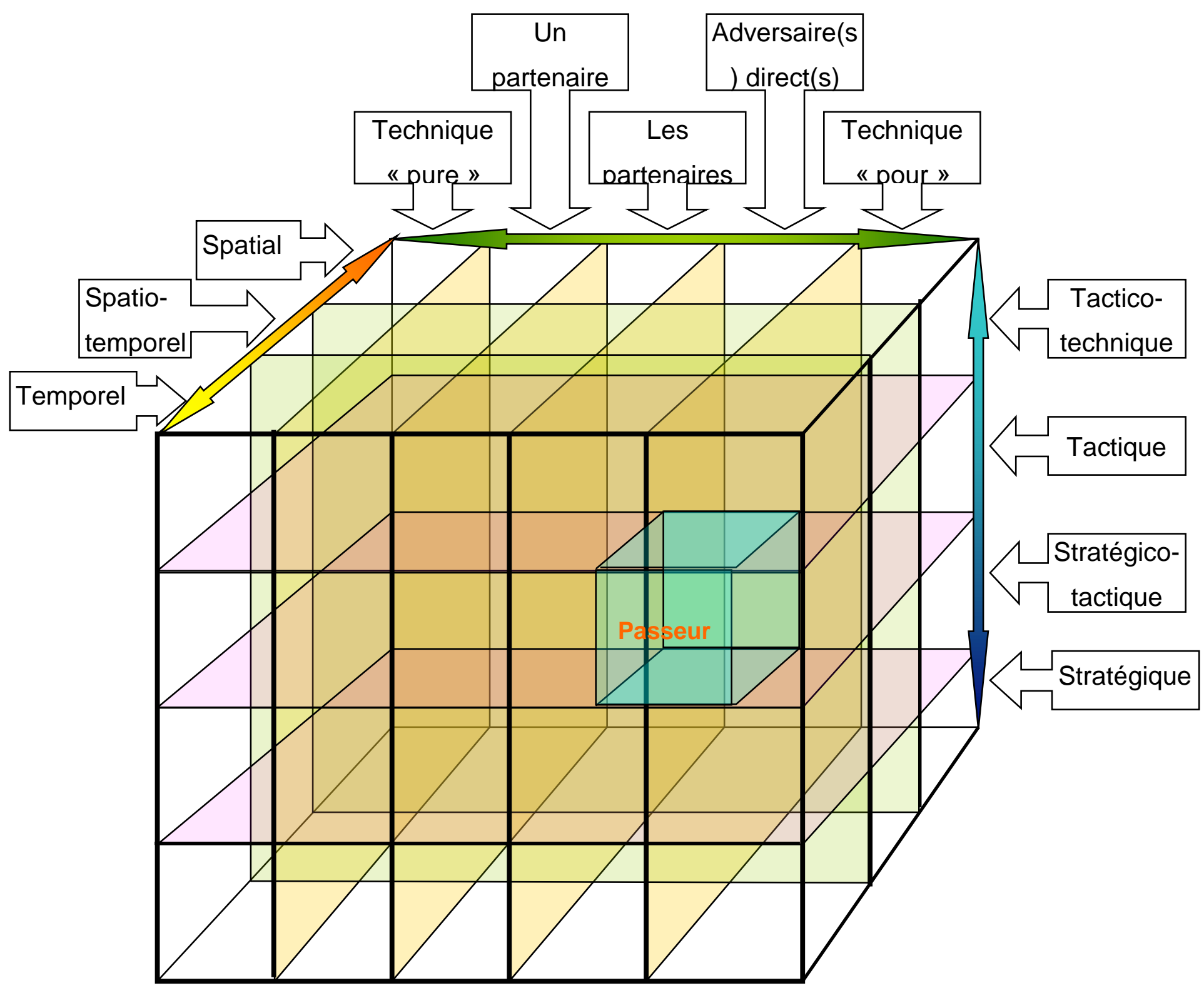

Figure 2. L'espace du genre du passeur de volley-ball est délimité par 3 axes 
Notre objectif a donc été de rendre visible cette contribution stylistique personnelle du passeur. Pour cela, nous avons structuré un référentiel en trois dimensions constitué par les trois axes représentatifs de l'activité de ces joueurs. Ainsi, le volume matérialisé représente le genre du «passeur de volley-ball». Au sein de ce volume, il est alors possible de définir les particularités stylistiques de notre passeur en le positionnant sur chacun des trois axes. Son style se trouve donc matérialisé par son emplacement au sein du volume représentant le genre.

Partant de l'analyse technologique, nous avons construit ces catégories pour pouvoir confronter, comparer, mesurer les différences d'interprétation d'une situation. II se trouve que le développement de l'outil d'analyse a montré des convergences tout à fait prégnantes entre les différents points de vue (analyse de l'activité, analyse technologique, analyse des entretiens). Ainsi, comme nous l'avons indiqué précédemment, une forte concordance de nature s'est révélée entre les trois axes d'analyse de l'activité décisionnelle du passeur et la triade de l'activité dirigée. Nous voyons dans cette concordance un argument en faveur de la validité du cadre d'analyse des entretiens. La mise en perspective des axes et de la triade de l'activité peut se matérialiser sous cette forme :
- Axe spatio-temporel
$\Leftrightarrow \quad$ Objet de la tâche

C'est ce que doit gérer réellement le passeur. D'une part, il est à l'origine du choix des combinaisons d'attaques. II doit donc organiser les alternatives d'attaques de son équipe en prenant en compte cette double dimension. D'autre part, il décide in fine quel est l'attaquant qui, parmi l'ensemble des alternatives possibles, sera le destinataire de sa passe.

- Axe stratégie-tactique $\Leftrightarrow$ Rapport aux autres

Ces autres sont aussi bien les partenaires que les adversaires. La prise en compte des uns comme des autres relève de préoccupations stratégico-tactiques. Ce sont les moments où ces focalisations sont réalisées qui déterminent du caractère plutôt stratégique ou plutôt tactique de l'action.

- Axe technico-technique $\Leftrightarrow$ Rapport au sujet lui-même

Ici, c'est du rapport au passeur lui-même qu'il s'agit. C'est le point ultime de son activité. C'est l'expression des autres données qui se réalise sous la forme de la manipulation de son outil (ses mains). Clot évoquerait le concept de catachrèse (Clot \& Gori, 2003) pour définir toutes les formes possibles d'utilisation de cet « outil ». Outil pour passer, accélérer ou conserver le ballon. Outil pour démarquer, fixer, feinter ou surprendre l'adversaire. 


\section{Résultats}

Les résultats qui suivent ne sont pas constitués de données numériques, de pourcentages ou de tests statistiques. II s'agit ici d'analyser des données qualitatives. Le travail d'analyse des résultats consiste à positionner sur chaque axe le passeur en prenant en compte ce qu'il nous a dit lors de l'entretien. Nous allons donc, à l'aide des verbatim, justifier de chacun de ces positionnements. Les résultats présentés sont issus d'un entretien réalisé avec un passeur de haut niveau. Nous le nommerons Jules.

4. 1. Contexte de l'action positive de Jules

C'est la demi-finale du championnat de France de Pro A. II s'agit d'un match de play-off contre l'équipe de Paris. Le public est important et fervent. II y a du bruit. L'équipe de Jules joue à domicile. À ce moment du match, l'équipe n'est pas au mieux. Jules exprime la nécessité de faire un coup qui redonne le moral à son équipe.
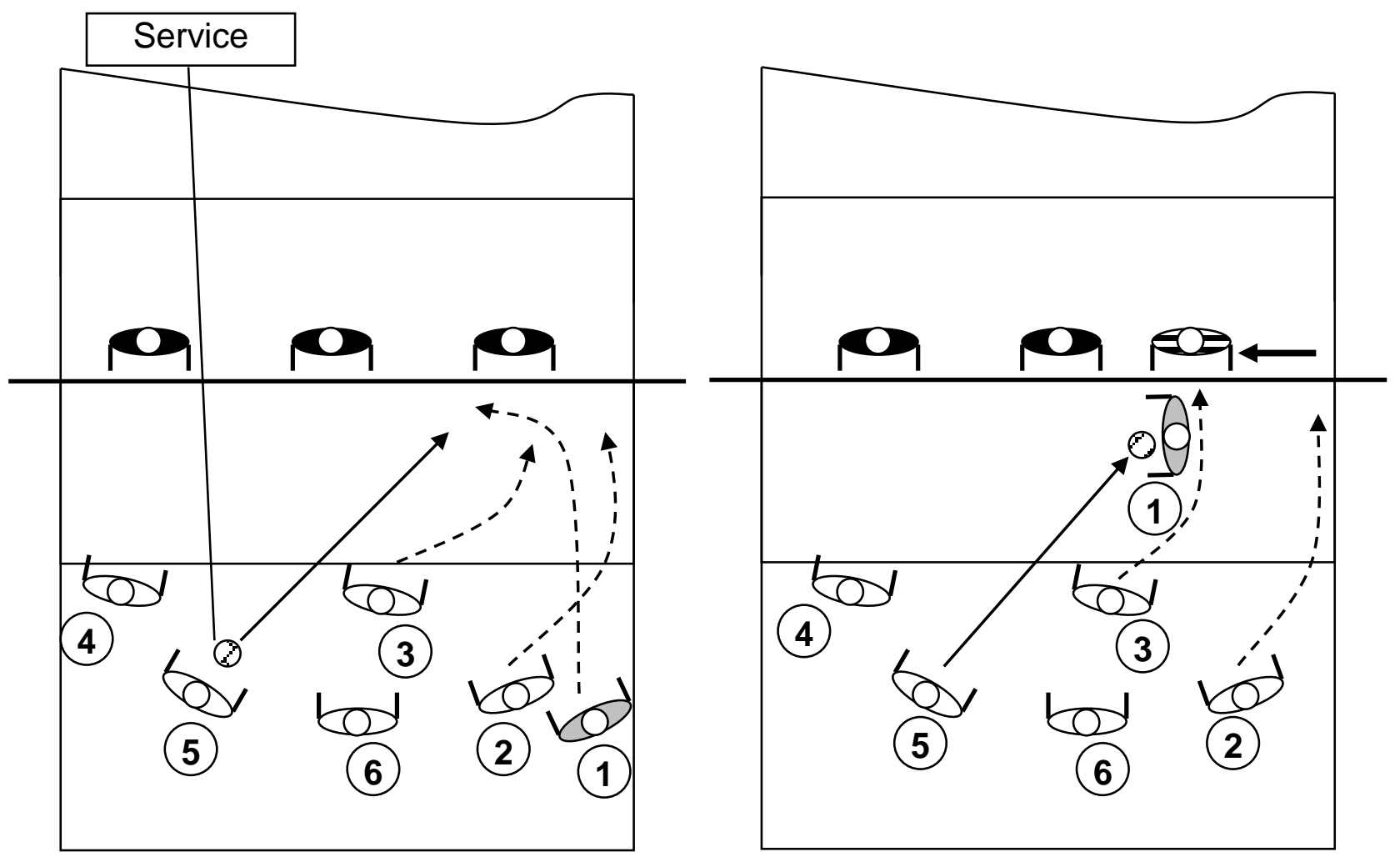

Figure 3 : Action positive de Jules. Sur le schéma de gauche, le service est parfaitement réceptionné par le joueur 5 . Le ballon arrive précisément sur le passeur. Le schéma de droite montre comment l'appel de balle du joueur 3 attire le contreur ailier (rayé) libérant l'espace d'attaque pour le joueur 2

4. 1. 1. Positions des joueurs (action positive)Jules est en poste 1 (arrière droit) au moment du service de l'adversaire. II pénètre sur le réceptionneur qui est en poste 2. II va devoir s'élancer à la frappe de balle du serveur pour se positionner prêt du filet afin de recevoir la réception et faire sa passe. Il annonce une combinaison en surcharge (courte 
arrière - demie derrière). Le réceptionneur est en poste 5. Le pointu est en poste 4 . Le central vient du poste 3. La balle est attaquée en poste 2. Le positionnement des adversaires est peu évoqué du fait que le choix de passe est établi avant. Jules sait à qui il va donner le ballon avant le service. II veut faire basculer la nervosité de son équipe en euphorie.

4. 1. 2. Mise en place du rapport d'opposition (action positive)

La réception qui arrive du poste 5 est parfaite. Jules sait qu'il va donner la balle à son réceptionneur-attaquant du poste 2. II va manipuler le contreur d'aile adverse pour lui faire croire qu'il va servir son central en courte arrière. II donne la balle en demie arrière pour son attaquant qui est démarqué.

4. 1. 3. Rapport de force des lignes au filet (action positive)

Jules est arrière et pénètre du poste 1 . II dispose donc de 3 attaquants au filet. II a annoncé une combinaison et a fait son choix de passe avant le service de l'adversaire. Tout le jeu consiste à fixer le contreur d'aile qui se trouve au poste 4 adverse (joueur rayé blanc et noir sur le schéma). II va donc s'employer à rendre suffisamment crédible l'éventualité de la courte arrière afin d'engager cet adversaire dans une action de contre de premier temps (contre d'entraide). De ce fait le joueur du poste 2 de l'équipe de Jules va se retrouver seul à l'attaque.

4. 2. Contexte de l'action négative de Jules

4. 2. 1. Positions des joueurs (action négative)

C'est le dernier match de la phase régulière du championnat. L'équipe de Jules joue à l'extérieur. II faut absolument gagner pour être dans les quatre premiers. La réception est envoyée vers le poste 2 et un peu dans le filet ce qui fait reculer Jules. Pour des raisons d'ordre psychologique, Jules a décidé avant l'échange de donner la balle à son pointu (attaquant de pointe). Jules a la possibilité de donner une fixation, une balle accélérée en poste 4 et une balle demie aux 3 mètres poste 1 . Malgré une réception d'une qualité moyenne, Jules peut encore donner la balle aux trois joueurs. Son recul vers le poste 2 attire le contreur central de ce côté du terrain. La programmation de la décision semble être lue par les contreurs adverses qui se préparent à intervenir. 

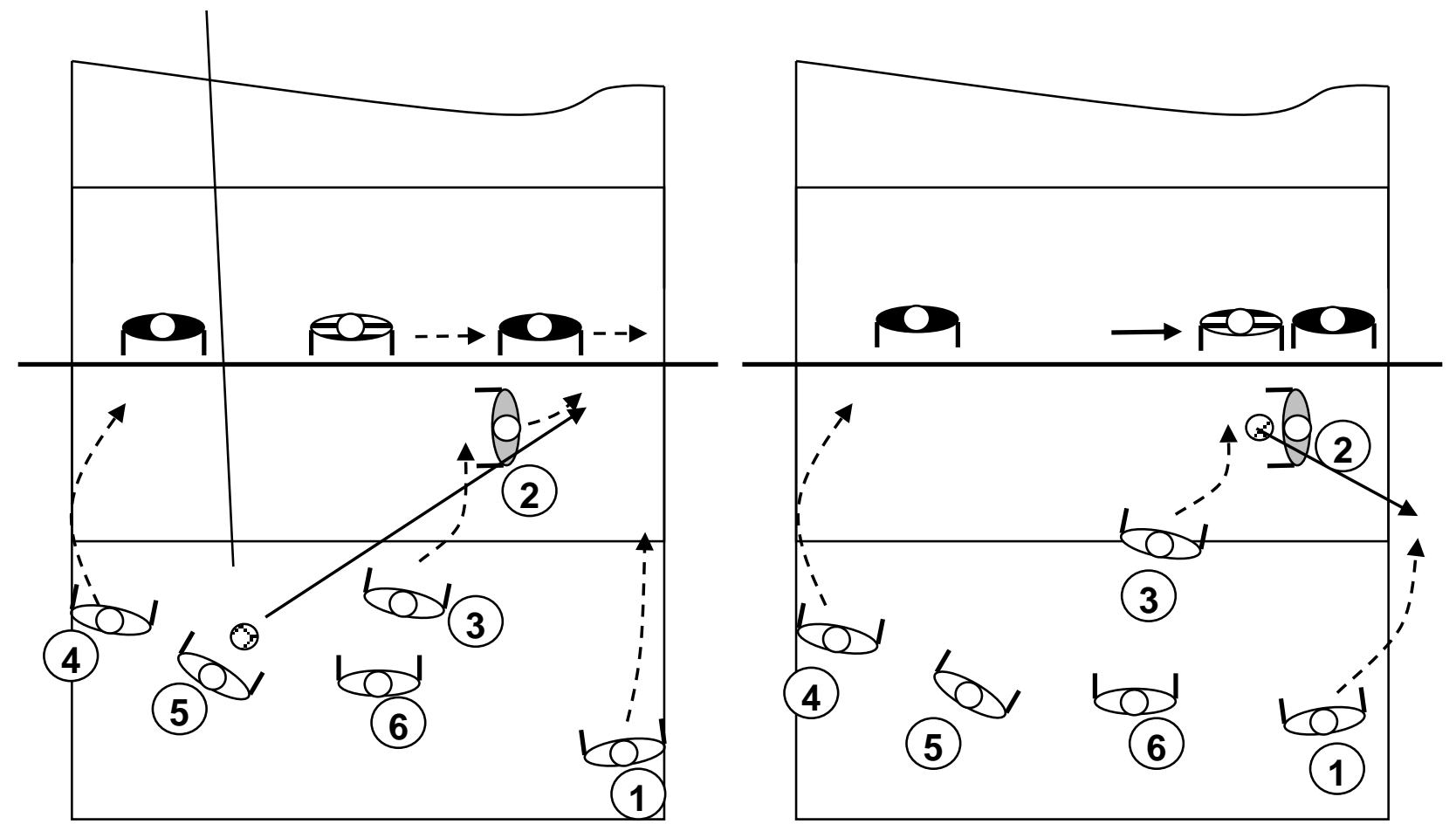

Figure 4 : Action négative de Jules. Sur le schéma de gauche, la réception médiocre du joueur 5 décale Jules vers l'arrière. De ce fait le contreur central (joueur rayé) se trouve attiré dans le même sens. Jules troublé par cette situation délivre une mauvaise passe. Son pointu se retrouve non seulement devant deux contreurs mais en plus, il doit attaquer une passe qui fuit en dehors du terrain.

\section{2. 2. Mise en place du rapport d'opposition (action négative)}

Il n'y a pas d'indication concernant la position exacte de l'équipe mais l'on sait que le pointu est arrière. Puisque le passeur joue en opposition avec l'attaquant de pointe, on déduit que Jules est avant. La réception est poussée vers le poste 2 ce qui fait reculer Jules. Il offre une passe de piètre qualité à son pointu qui attaque dans le filet.

\section{2. 3. Rapport de force des lignes au filet (action négative)}

Jules est avant mais il dispose de trois attaquants. Le central (joueur $n^{\circ} 3$ ) de Jules se déplace vers lui pour venir en fixation. Ce mouvement du joueur de centre attire les contreurs adverses qui se retrouvent à deux face au poste 2 . Comme il a décidé de donner la balle à son pointu avant l'échange, mais qu'il se rend compte que tactiquement ce n'est pas une bonne option, Jules est perturbé. Cette remise en cause de l'option initiale en cours de jeu le trouble au point de manquer la réalisation technique de sa passe. Son pointu se retrouve non seulement devant deux contreurs qui l'attendent de pieds fermes, mais en plus, il doit attaquer une passe qui fuit hors des 3 mètres. Le joueur 
de pointe ne peut même pas attaquer correctement le ballon et tombe en arrière et au sol. Le ballon finit dans le filet.

\section{3. Positionnement de Jules sur les trois axes}

Pour chacun des axes, le discours est réorganisé. II s'agit de retrouver les passages du texte qui ont trait à l'une des trois catégories. L'étude des indices dévoilés par le passeur lors de l'entretien permet ainsi de positionner le passeur sur chaque axe.

\section{3. 1. Positionnement de Jules sur l'axe spatio-temporel}

L'action positive décrite par Jules est une action typique de surcharge. L'idée consiste à produire un appel de balle en temps rapide dans un intervalle entre deux joueurs. Jules en obligeant le contreur d'aile à venir aider son centrale (entraide) libère cet espace pour un autre de ses attaquants. Toute la difficulté de ce schéma tactique consiste à attirer ce contreur d'aile, à rendre crédible cette attaque dans l'intervalle. Pour Jules, la solution du problème tourne autour du temps de passe. C'est comme si une temporalité parallèle se créait. II y a le temps qui s'écoule normalement et puis un temps hypertrophié, qui se déroule lentement. Jules nous décrit bien l'action comme au ralenti.

Interviewer : Et à quel moment tu fais ton impulsion là, par rapport à ça ? Tu es dans l'action, elle arrive là ...

Jules : C'est au moment où elle (la balle) commence à descendre je pense.

Interviewer : Au moment où elle commence à descendre ?

Jules : Euh... un peu plus tard, plus tard.

Interviewer: Un peu plus tard?

Jules : Ouais

Le temps est perçu comme étiré. Le moment où la balle commence à descendre semble relativement précis mais Jules corrige. Un peu plus tard, plus tard dit-il.

Interviewer : Donc tu déclenches et tes bras ils vont où là ?

Jules : Là, ils sont ... tendus le plus possible.

Interviewer : Tendus, ouais, et comment ça se passe alors. Tendus par rapport à la rencontre avec la balle?

Jules : Mais en fait, le but du jeu là, sur cette action là, c'est de faire croire qu'on va toucher la balle à un moment donné et en fait la toucher ici (il montre avec ses bras).

L'interviewer parvient à lui faire déplier l'action avec une granularité assez fine.

Interviewer : Qu'est-ce qui rentre en contact avec la balle ?

Jules: Les pouces surtout

360 Interviewer : Surtout les pouces?

Jules : Ouais 
Interviewer : Et là tu fais quoi ?

Jules : Je mets juste un petit coup.

Interviewer : Mais tu avais tes bras tendus ?

Jules : Ouais, ouais mais j'ai les bras tendus mais en fait, au moment de rentrer en contact avec la balle, je descends un peu, et je mets un petit coup de pouce.

Interviewer : Et tu fais tout ça en même temps ?

Jules : Ouais, c'est très court quoi, c'est çà, et en fait, c'est limite même je les tends un peu plus juste avant de la toucher et je les re-détends pour faire croire encore plus ...

Ici, le temps s'exprime dans des grandeurs imperceptibles. La qualité de la réception qui arrive parfaitement permet un saut très précoce ce qui a pour conséquence de créer un espace de temps à l'intérieur duquel Jules va pouvoir travailler. On perçoit donc une focalisation sur la dimension temporelle.

Examinons maintenant le cas de l'action négative. Rappelons que dans cette phase de jeu, Jules est mis en difficulté par une réception qui arrive derrière lui et le contraint à reculer. II a décidé avant le service adverse de donner la balle à son pointu. II nous semble que tout est dit dans l'extrait qui suit :

Interviewer : Donc là, tu savais à qui tu allais la donner?

Jules : Je savais à qui je la donnais

Interviewer: Tu es en train de monter tes bras pour la donner

Jules : Voilà. Et en fait au moment où ... si... si au moment où j'hésite... si au moment où je fais la passe j'hésite, je suis mort.

Ici, contrairement à l'exemple précédent, la réception n'est pas parfaite ce qui conduit les contreurs adverses à un repositionnement qui n'était pas prévu. Ce changement est perçu par Jules qui du coup doute de son option initiale. L'espace temporelle se remplit alors d'une surcharge cognitive qui empiète sur le temps disponible. Ce dilemme est bien exprimé dans ce qui suit :

Jules : Et donc en plus, ça me pénalise sur la précision de la passe parce que au moment où je sais, je n'ai pas... je ne suis pas relâché comme il faudrait que je sois, et j'ai un petit doute qui s'insère, ce qui fait que je vais là louper en fait, c'est un cercle vicieux.

C'est donc ce doute qui perturbe l'organisation du mouvement. II ne monte plus assez les bras (en a-t-il le temps ?) et de ce fait, il ne prend pas la balle assez haute. II révèle son mode d'organisation par cette phrase :

Jules : Ben en fait, le truc c'est que quand je fais la passe, pour moi hein, pour moi, plus je sais tôt à qui je vais la donner, et plus belle je vais mettre la passe. 
Encore une fois, le temps est au cœur de l'organisation de Jules. Plus je sais tôt et plus la passe sera belle.

L'analyse de ces deux actions de jeu révèle que Jules est très dépendant du temps. On pourrait dire pour être plus précis, qu'il doit pouvoir organiser, ordonner son temps. Soit il peut déployer son action selon une temporalité très fine et l'action est positive. Soit pour des raisons qui lui sont indépendantes, il ne peut plus gérer cet intervalle temporel et l'action tourne mal. Ce constat nous amène à placer Jules sur la position temporelle de l'axe spatio-temporel.

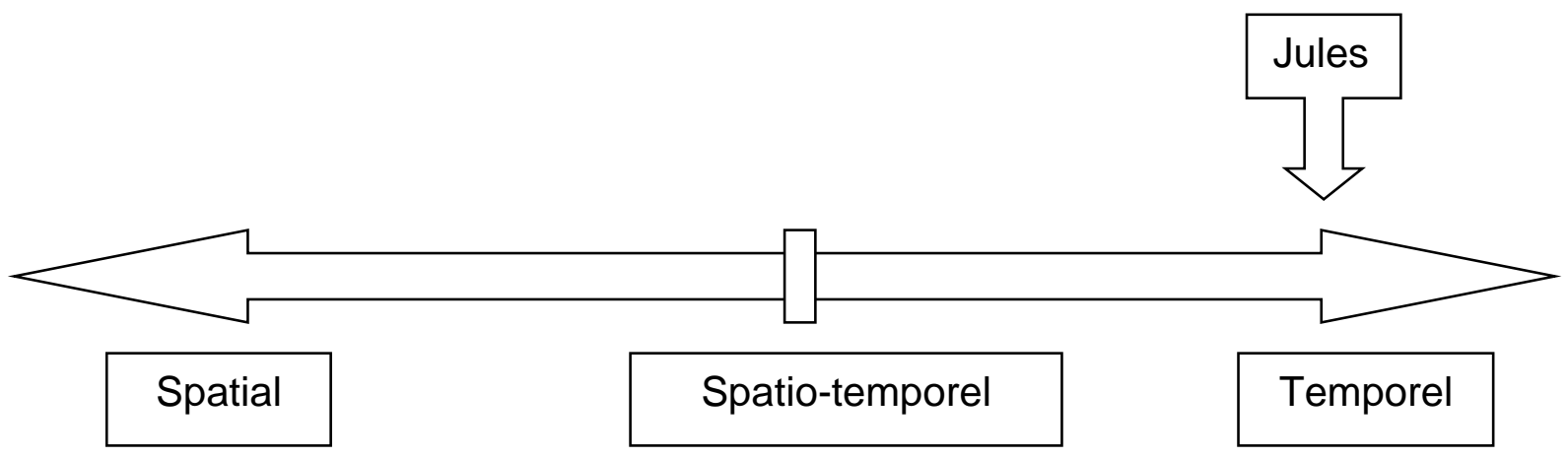

Figure 5. Position de Jules sur l'axe spatio-temporel.

4. 3. 2. Positionnement de Jules sur l'axe stratégico-tactique

Comme nous avons pu le constater dans l'analyse de l'axe précédent, Jules décide beaucoup à l'avance. Malgré tout, il reste très réceptif à ce qui peut se dérouler sur son terrain. Son mode d'anticipation nous est livré dans ce qui suit:

Jules : En fait, avant chaque point, j'essaie le plus souvent possible, de décider plusieurs alternatives. S'il il y a bonne réception je fais plutôt ça, s'il y a mauvaise réception je fais plutôt ça.

Si nous voulons préciser encore la façon dont il décide de telles ou telles alternatives, nous devons tenter de relever dans son entretien comment il prend en compte l'adversaire. Or, nous n'avons que très peu de référence à l'adversaire. II y a une combinaison annoncée avant le service mais les témoignages ne font pas état d'un mode d'organisation qui fasse référence aux spécificités de la ligne adverse. Ce sont toujours les partenaires qui déterminent le choix fixé a priori. Soit que tel attaquant n'est pas au mieux, soit que celui-là doit être remis en jeu. II procède à une analyse précoce de la situation, et se focalise ensuite sur la réussite de l'action. II oublie alors les adversaires, voire les partenaires pour se concentrer sur son action et le ballon comme il l'exprime pour son action positive: 
Interviewer : Non, à quoi tu es attentif au moment où tu pars ?

Jules : À la balle, à la balle ...

Interviewer: Tu vois la balle ?

Jules : Ouais, je vois la balle c'est tout. Je me suis fermé complètement par rapport au reste et je regarde que la balle.

L'action se déroule ensuite sans interférence. C'est comme si lui-même s'interdisait de penser à autre chose. Puis une fois la balle sortie de ses mains Jules espère que l'issue sera favorable :

Jules: ...C'est-à-dire que je suis resté bloqué sur le ballon en faisant attention à ma technique, et en me disant, en espérant que le gars allait se laisser prendre.

II revient sur l'organisation de son action

Jules : Ouais, là, j'ai oublié le central et j'ai oublié les bloqueurs et je fais plus attention à ça sachant que à partir du moment où j'ai vu que c'était une bonne réception, je savais ce que j'allais faire, ça ne m'arrive pas tout le temps, mais à cet instant précis là, j'avais décidé ça.

Jules a décidé d'une combinaison parce qu'elle marche d'habitude. Ensuite il se coupe du monde et tente de faire une réalisation la plus parfaite possible. L'analyse de l'action négative ne fait que confirmer ce que nous avons pu percevoir dans l'action positive. Ici, la mise en difficulté que Jules subit et qui le fait reculer lui ouvre paradoxalement son champ de vision. Il a bien décidé à qui il devait donner la balle, mais son déplacement provoque un déplacement des contreurs adverses qu'il perçoit très clairement :

Jules : Et euh ... donc en fait je prends la balle, et je ne sais pas pourquoi, je lui donne, alors qu'il faut, que dans ce cas là, il ne faut vraiment pas que je lui donne. Parce qu'il y a le central en plus qui est juste devant moi qui m'a suivi, donc il y a le central adverse qui a suivi aussi.

La perception du déplacement du contreur adverse met le doute chez Jules quant à la pertinence de son choix initial. On le voit bien ici, l'action négative dévoile contradictoirement le mode de fonctionnement de ce passeur. Lorsque tout va bien, Jules joue par rapport à ce qu'il a prévu. Il a donc un mode d'organisation stratégico-tactique fortement orienté vers ses partenaires. II suffit que la situation l'amène à percevoir les mouvements de l'adversaire pour que l'espace temporel dédié à la réalisation du geste soit grignoté par les mécanismes de prises de décision. Cela atteste bien que Jules programme ses décisions avant que la balle ne soit en jeu et en référence à des schémas de jeu typiques (surcharge). Pour les raisons évoquées, nous placerons Jules sur la position stratégico-tactique de l'axe. 


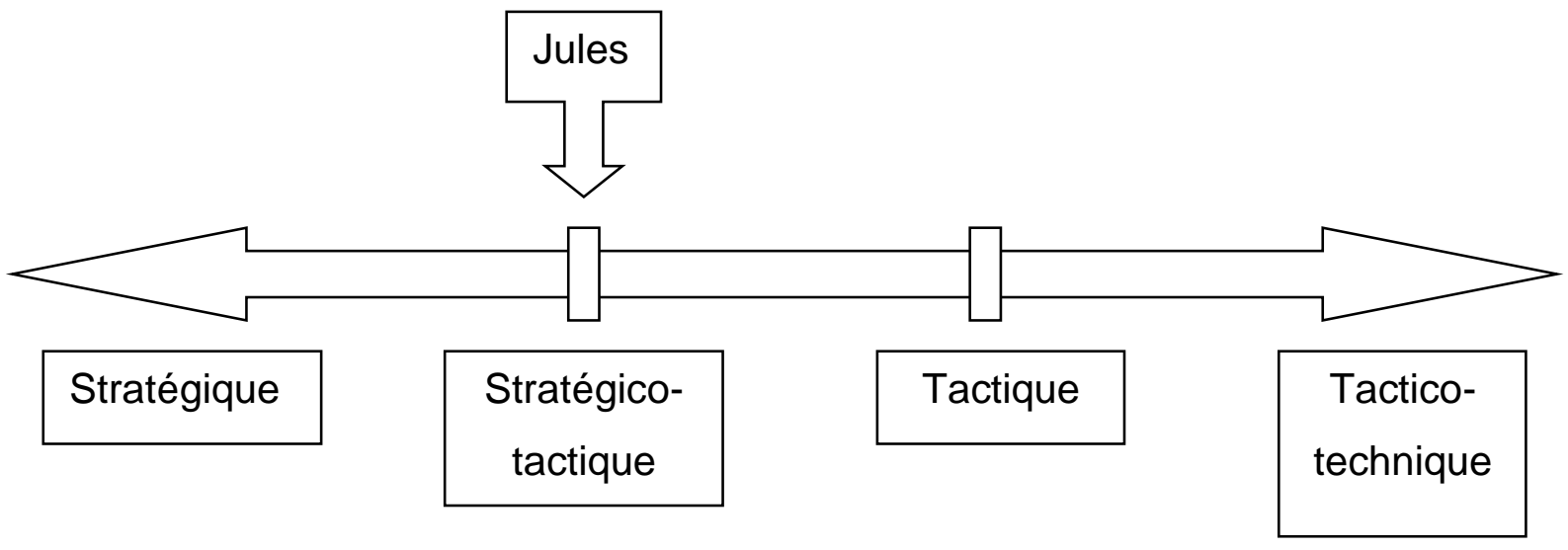

Figure 6. Position de Jules sur l'axe stratégico-tactique.

4. 3. 3. Positionnement de Jules sur l'axe technico-technique Dans la description de son action positive, Jules donne la tendance de son mode de relation à la technique. II se positionne radicalement dans la prise en compte différée de ses adversaires. II a décidé avant le service adverse le joueur qui aura le ballon. II faut maintenant cacher cette décision le plus longtemps possible (ligne 305) :

Jules :... voilà, donc il y avait bonne réception donc je n'avais plus qu'à faire attention à ma technique pour que le ... pour espérer que ça marche.

Comme libéré de la partie prise de décision, Jules peut se consacrer entièrement à la réalisation technique de son geste.

Jules : Mais en fait, le but du jeu là, sur cette action là, c'est de faire croire qu'on va toucher la balle à un moment donné est en fait la toucher ici.

Cette volonté de masquer son jeu se décrit très précisément. La technique s'exprime jusqu'au bout des pouces.

Jules : Ouais, c'est très court quoi, c'est ça, et en fait, c'est limite même je les tends un peu plus juste avant de la toucher et je les re-détends pour faire croire encore plus ...

Plus loin, à propos de l'action négative, on perçoit que ce temps de manipulation est réduit par le doute. Ici Jules exprime ses limites techniques lorsque la situation n'est pas conforme à ce qui était prévu. 
Jules : Et donc en plus, ça me pénalise sur la précision de la passe parce que au moment où je sais, je n'ai pas, je ne suis pas relâché comme il faudrait que je sois, et j'ai un petit doute qui s'insère, ce qui fait que je vais la louper en fait, c'est un cercle vicieux.

Le doute qui est dû à une situation non-conforme ne le libère pas pour se concentrer exclusivement sur sa technique de passe.

Jules : Et en fait, même si je ... peut-être que si je lui avais fait une bonne passe il aurait pu mettre le point. Mais en me disant, merde ce n'est pas là qu'il faut la donner, je deviens moins précis, je suis imprécis et je fais une mauvaise passe en plus.

C'est comme si la prise en compte de l'adversaire, suscitée par une situation non prévue (il doit reculer vers le poste 2), saturait « l'espace cognitif » de Jules ne laissant plus assez de place pour procéder aux opérations de manipulation du ballon. II prend conscience que le choix programmé n'est plus pertinent.

Jules : Je me rends compte que je suis près de la mire au poste 2 , que... que le central est venu avec moi, que a priori, je n'ai pas regardé mais a priori, il y a deux gars qui sont venus, deux bloqueurs qui sont venus.

Cette prise de conscience détériore ses capacités techniques. II parle de ses bras :

Jules : Disons qu'en fait ils sont en train de monter, mais ils ne finissent pas l'action, ils s'arrêtent un peu et après ils la finissent ce qui fait que ... ça fait une mauvaise passe quoi. II est donc nécessaire que Jules fasse abstraction de son environnement pour être le plus disponible possible. L'apparition d'un imprévu risque de tout désorganiser. II procède en deux temps. Un temps de prise de décision antérieur à l'échange et un temps de manipulation. En conséquence, il fait plutôt référence à la technique «pour». De fait, lorsque la situation est favorable, il a programmé son choix et tente de manipuler la situation ou le ballon « pour » masquer ses intentions et faire croire à ce qu'il ne réalisera pas.

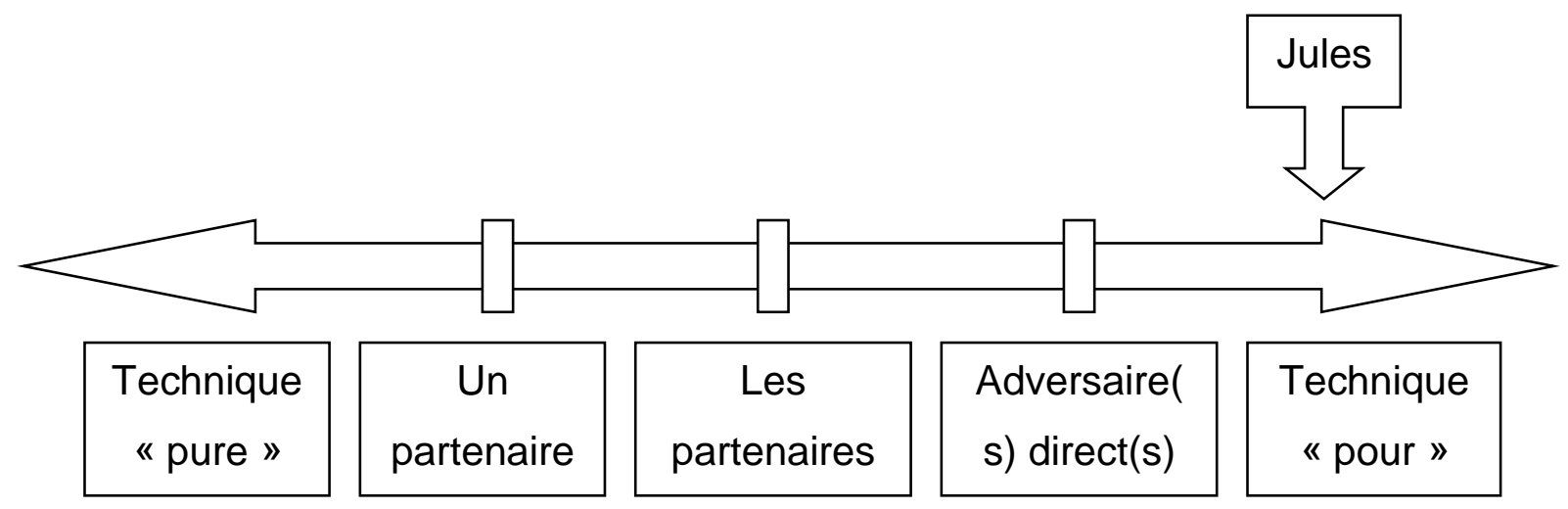

Figure 7 : Position de Jules sur l'axe technico-technique. 
4. 4. Détermination du style de Jules

Du positionnement de Jules sur chacun des axes, il ressort que :

Jules organise ses alternatives d'attaques en privilégiant la dimension « temporelle ».

Jules a un rapport aux autres fondé sur une approche « stratégico-tactique ».

Jules privilégie un mode de focalisation centré sur une «technique pour » leurrer ses adversaires.

Le style de Jules peut donc se matérialiser comme suit dans l'espace du genre.

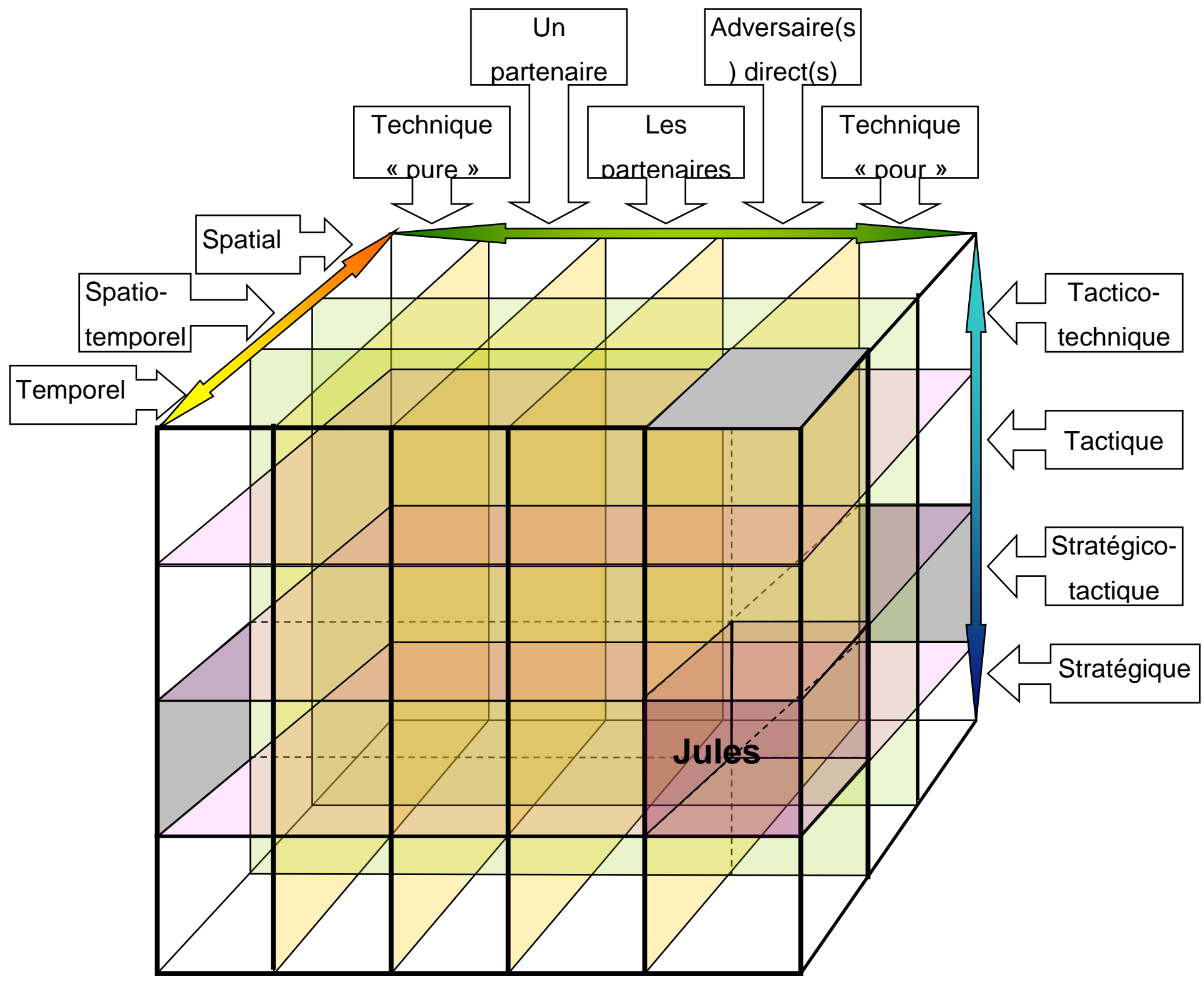

Figure 8. L'espace déterminé par les 3 axes représente le genre du « passeur de volleyball ».La position de Jules dans ce volume indique son style.

\section{Discussion}


En guise de conclusion, nous voulons discuter différents points relevant de la méthode, de l'analyse des résultats et de la définition du style du passeur.

5. 1. Sur la méthode

Nous l'avons évoqué plus haut, la méthode proposée ici fait référence à un cadre théorique multi référencé. L'entretien d'explicitation est-il compatible avec la clinique de l'activité ? Nous avons défendu l'hypothèse d'une indépendance des données recueillies et des méthodes qui les font naître. Nous avons souligné l'intérêt d'une stratégie expérimentale qui privilégiait l'étude des opérations à celui du développement de l'activité. II nous semble que notre différence de perspective par rapport à une clinique de l'activité justifie l'adaptation de la méthode. La collusion de l'analyse technologique et de la triade de l'activité nous semble être un argument en faveur de cette démarche.

Sur l'analyse des résultats

Comme pour chaque méthode nouvelle et non éprouvée, se pose le problème de la validité de la recherche. Peut-on réellement caractériser le style d'un passeur sur la base de deux actions de jeu? Pour répondre à cette objection légitime, nous voulons développer deux points. Le premier point concerne le contrat qui est passé entre l'interviewer et le passeur. Certes on peut toujours dire que deux actions ne sont pas suffisantes pour déterminer un style. Cependant ces deux actions ont été choisies par le passeur. Les consignes étaient de choisir des actions dans lesquelles il a été particulièrement à son avantage ou, a contrario, dans lesquelles il a été en grande difficulté. Nous pensons alors pouvoir faire raisonnablement l'hypothèse que lorsque l'on choisit deux actions dans un corpus disponibles de plusieurs centaines d'actions, cela n'est pas fait au hasard. II nous semble légitime de considérer que ces actions sont pleinement représentatives de ce que le passeur juge être une action positive ou négative. Le deuxième point concerne ce que nous avons appelé la méthode des contrastes. II se trouve que dans les différents cas étudiés par ailleurs, l'action négative corrobore toujours les données de l'action positive. Pour Jules, si la réception est parfaite, il peut faire abstraction de son environnement pour s'adonner à la manipulation technique de son adversaire. Si au contraire la situation n'est pas optimale, les informations extérieures le troublent au point de contrarier sa dextérité. Le négatif vient donc corroborer le positif et consolide ainsi les résultats issus de l'analyse de l'action positive.

5. 2. Sur la définition du style

Le style ne peut se définir que par rapport à un genre. Nous voulions rendre visible l'expression stylistique de notre passeur Jules. Le parallélépipède est une représentation du genre et la position de passeur dans cette espace à trois dimensions une 
représentation du style. Mais les styles sont une réinterprétation du genre. Si lors d'études postérieures, d'autres passeurs montrent des stratégies différentes pour gérer la distribution du jeu, l'espace du genre sera modifié et de nouveaux styles apparaîtront. II n'est donc pas fait référence à une conception figée du genre et du style dans cette étude. En revanche, nous voulons utiliser ultérieurement cet outil pour comparer les passeurs entre eux. Conscient que le style est une recomposition personnelle du genre, il nous semble que nous pourrons faire apparaître le style de différents passeurs dans l'espace du genre provisoirement défini.

En conclusion, cette recherche a permis de faire une description, un instantané de la réalité des modes de gestion de la distribution du jeu d'un passeur de volley-ball. Elle montre que limiter son rôle à la simple relation passeur-attaquant n'est pas suffisant. De la même façon, dans la formation, la problématique de la technique spécifique doit être réexaminée. II nous semble qu'elle doit se construire dialectiquement avec le projet stratégico-tactique du joueur. Bien sur, le cas de Jules n'est qu'un cas unique. II nous faut donc étendre cette analyse à d'autres passeurs de haut niveau pour déterminer s'il existe une disparité ou une certaine homogénéité des styles. II reste que l'outil construit offre la perspective de son utilisation systématique pour permettre de telles comparaisons.

\section{Bibliographie}

Barbier, J.-M. (2000). L'analyse de la singularité de l'action. Paris: PUF.

Bonnefoy, G., Lahuppe, H., \& Né, R. (1997). Sports collectifs. Jouer en équipes. Hand-ball Volley-ball: Les cahiers ACTIO.

Clot, Y. (1999). La fonction psychologique du travail. Paris: PUF.

Clot, Y. (2000). Analyse psychologique du travail et singularité de l'action. In CNAM (Ed.), L'analyse de la singularité de l'action. PUF.

Clot, Y. (2004). Action et connaissance en clinique de l'activité. @ctivité, 1(1), 23-33.

Clot, Y., \& Faïta, D. (2000). Genre et style en analyse du travail. Travailler, 4, 7-42.

Clot, Y., Faïta, D., Fernandez, G., \& Scheller, L. (2002). Entretiens en autoconfrontation croisée: une méthode en clinique de l'activité. Education Permanente, 146, 17-27.

Clot, Y., \& Gori, R. (2003). Catchrèse : éloge du détournement. Nancy : Presses Universitaires de Nancy.

Coulibaly, Z. (1984). Essai d'analyse de la prise d'information visuelle chez le passeur en volley-ball (Mémoire INSEP).

Coutau-Bégarie, H. (2003). Traité de stratégie (4 ed.): Economica. 
Deleplace, R. (1983). La recherche sur la spécialisation, l'entraînement, la performance, La recherche en STAPS, Nice 19-20 sept. 1983.

Éloi, S. (2001). Le rôle du passeur. Actes du colloque des entraîneurs de volley-ball. Paris, 16-17 juin 2001.

Éloi, S., \& Uhlrich, G. (2001). Contribution à la caractérisation des sports collectifs : les exemples du volley-ball et du rugby. STAPS, 56, 109-125.

FIVB. (2005). Règles officielles du volley-ball: Fédération Internationale de Volley Ball.

Gouju, J.-L. (2002). L'action comme fenêtre attentionnelle? Expliciter, 44, 1-9.

Gouju, J.-L. (2005). Documenter les aspects sensibles de l'expérience: l'approche de la psychophénoménologie. Expliciter, 58, 16-26.

Le Moigne, J.-L. (1999). La modélisation des systèmes complexes (2 ed.). Paris: Dunod.

Léontiev, A. (1972). Le développement du psychisme. Paris: Edition sociales.

Macquet, A.-C. (2001). Le contrôle des situations chez les volleyeurs experts : informations perçues et connaissances mobilisées ou construites. Thèse de Doctorat. Université Paris XI. Non publiée.

Morin, E., \& Le Moigne, J.-L. (1999). L'intelligence de la complexité. L'Harmattan.

Mouchet, A. (2003). Un nouveau regard sur les décisions tactiques des joueurs de rugby. Expliciter, 48, 1-15.

Mouchet, A. (2005). Modélisation de la complexité des décisions tactiques en rugby. eJRIEPS, 7, 3-19.

Pastré, P. (2002). L'analyse du travail en didactique professionnelle. Revue Française de Pédagogie, 138, 9-17.

Récopé, M. (1996). Statut et fonction du schème de duel dans l'organisation de l'action motrice d'opposition: le cas du Volley-Bal. Thèse de doctorat. Université René Descartes, Paris V. Non publiée.

Récopé, M. (2002). Implications didactiques d'une théorie de l'action motrice: synthèse assimilatrice et enseignement fonctionnel du Volley-Ball. Impulsions, 3, 27-62.

Rosinski, H. (1950). Frontières conceptuelles entre stratégie, opérations et tactique dans l'Art de la guerre, Étude inédite. Traduit de l'anglais par Jean et Bernadette PAGES.

Schmitt, J.-M. (1996). Le passeur. Sur Cassette VHS: Production FFVB / CREPS AlpesVivarais.

Simonet, P., Hippolyte, R., \& Petit, D. (1990). La formation du passeur. Sur Cassette VHS: Production INSEP.

Vermersch, P. (1994). L'entretien d'explicitation. Paris: ESF Éditeur. 
Vermersch, P. (1999). Pour une psychologie phénoménologique. Psychologie Française, 44(1), 7-18.

Vermersch, P. (2002a). De l'explicitation au point de vue en première personne. Impulsions, 3, 93-121.

Vermersch, P. (2002b). Quelques études de cas sur l'articulation entre situation d'étude et développements théoriques. Expliciter, 45, 1-11.

Vermersch, P., \& Maurel, M. (1997). Pratiques de l'entretien d'explicitation. Paris: ESF. 